\title{
Hematological and Biological Changes in Different Routes of Experimental Trypanosoma Brucei Infection in Albino Rats
}

\author{
Ameh MP ${ }^{1}$, Jatau ID ${ }^{2}$, Ada G $^{1}$ and Akefe I0*3 \\ ${ }^{1}$ Department of Veterinary Pharmacology and Toxicology, Ahmadu Bello University, Zaria, Nigeria \\ ${ }^{2}$ Department of Parasitology and Entomology, Ahmadu Bello University, Zaria, Nigeria \\ ${ }^{3}$ Department of Physiology, Biochemistry and Pharmacology, Faculty of Veterinary Medicine, University of Jos, Nigeria
}

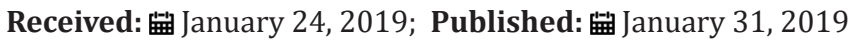

*Corresponding author: Akefe IO, Department of Physiology, Biochemistry and Pharmacology, Faculty of Veterinary Medicine, University of Jos, Nigeria

\begin{abstract}
The present study was aimed at investigating the Hematological and biological changes in different routes of Trypanosoma brucei infection in Albino rat. Five groups of four Albino rats, each of which was challenged with approximately $1.6 \mathrm{X} 10^{6}$ Trypanosoma brucei, except for the fifth group which served as uninfected control. The routes of inoculation were; Intravenous (IV), Intramuscular (IM), Subcutaneous (SC) and Intraperitoneal (IP). The rats were monitored for prepotency, parasitemia, changes in Packed Cell Volume (PCV), total leukocyte count, plasma protein, mortality and relapse during the 21 days course of infection. The result revealed that all the infected rats developed parasitemia, with mean pre-patent periods of $3.00 \pm 0.000,4.00 \pm 1.000,7.50 \pm 1.500$ and $9.00 \pm 1.732$ days respectively, for IV, IM, SC and IP routes. There was decreased PCV in all the infected groups compared with the uninfected control which was not significant $(\mathrm{P}<0.05)$ except for the IP group. Leukocytosis was most significant in the IP group compared to other groups. The mean total plasma protein increased from day zero through day 12 in all the routes of inoculation, but there was no significant difference between all the groups. The mean parasitemia increased progressively in IV route compared with other groups, but least in the IP route group. The mean survival time of the infected rats were $15.25 \pm 2.016,13.25 \pm 2.720,12.25 \pm 0.250$ and $12.75 \pm 1.250$ days for IV, IM, SC and IP route, respectively. In conclusion, different routes of inoculation of Trypanosoma brucei showed significant variation in pre-patency, parasitemia survival time but not in PCV and Total plasma protein.
\end{abstract}

Keywords: Hematology; Trypanosoma brucei; Parasitemia; Packed Cell Volume

Abbreviations: PCV: Packed Cell Volume; WHO: World Health Organization; CSF: Cerebrospinal Fluid; HCT: Haematocrit Centrifugation Technique; WMT: Wet Mount Technique; TBS: Thin Blood Smear; TPP: Total plasma Protein; EDTA: Ethylene Diamine Tetraacetate; WBC: White blood cell

\section{Introduction}

Trypanosomosis is an infectious disease caused by pathogenic blood parasites known as trypanosomes (Trypanosoma spp) and transmitted through tsetse flies (Glossina spp). The disease is unique to Africa, affecting both human (human African trypanosomosis or sleeping sickness) and animals (animal African trypanosomosis or Nagana). It occurs in 37 sub Saharan countries, covering more than 9 million square kilometres; an area that corresponds approximately to one third of Africa total land mass [1]. Trypanosomosis is recognized as one of the major factors limiting livestock production in Africa especially in the sub-Saharan region [2]. The effects of this disease are recognized by the World Health Organization (WHO) and several programs and campaigns have been formed to eradicate this disease [3]. Nevertheless, this disease remains one of the most regulated tropical diseases and steps should be taken to prevent transmission of the disease and treat infected individuals with better treatment options [4]. Trypanosomes are unicellular protozoans of the family Trypanosomatidae that are transmitted by their only vector, the tsetse fly. The tsetse fly is of the genus Glossina and many different species of this fly exists which can transmit the trypanosomes [5]. The fly is unique in that it is viviparous (gives live birth of a developed larva) and mates only once in its life, both of which have important implications in its eradication strategies (WHO).

The two different strains of the same species (gambiense and rhodesiense) infect human populations only but are found in slightly different habitats and reservoirs. The gambiense strain is an 
anthroponotic strain causing the chronic form of sleeping sickness that transmits from human-to-human and humans' function as the main reservoir [5]. Transmission of the trypanosome begins with the tsetse fly bite that develops an inflammation of the skin known as a chancre. This allows the fly to transmit the metacyclic trypanosomes [6]. Pathogenesis within the human body host is divided in to two stages: the haemolymphatic stage followed by the meningoencephalitic stage. In the haemolymphatic stage, the trypanosome will then enter the lymphatic vessels and bloodstream of the human host [6]. Subsequently in the meningoencephalitic stage, the trypanosome invades the connective tissues and later, the choroid plexus into the brain and Cerebrospinal Fluid (CSF) [6]. The trypanosome transmitted exists in its metacyclic stage where it will undergo binary fission every six hours to form slender forms of the protozoan [6]. Infection of the host only occurs when the protozoan exists in its slender form but can alternate with the nonproliferative stumpy form to transmit back to the vector [6].

\section{Materials and Method}

\section{Experimental Animals}

Twenty adult rats (Rattus norvegicus) were purchased from the animal house of the Department of Veterinary Parasitology and Entomology, Faculty of Veterinary Medicine, Ahmadu Bello University, Zaria, Nigeria for the experiment. The rats were housed in a standard rat cage at the experimental animal house of the Department of Veterinary Parasitology and Entomology, Faculty of Veterinary Medicine, Ahmadu Bello University, Zaria. The animals were allowed to acclimatize for one week during which they were screened for haemoparasitic infections. They were fed throughout the experiment with self-made grower mash and water was supplied ad libitum.

\section{The Parasites}

The parasite used was Trypanosoma brucei which was obtained from the Department of Parasitology and Entomology, Faculty of Veterinary Medicine, Ahmadu Bello University, Zaria, Nigeria. The parasites were propagated in laboratory rats by intraperitoneal inoculation and monitored until the development of parasitaemia of up to 20-30 parasites per field on wet mount.

\section{Experimental Design}

The rats were grouped into five groups based on routes of inoculation (Intravenous IV, Intramuscular IM, Subcutaneous SC, Intraperitoneal IP) and uninfected control UC. Each group contain four rats tagged 1-4 namely; IV1-IV4, IM1-IM4, SC1-SC4, IP1-IP4 and then uninfected control UC1-UC4. Prior to the experimental infection, the rats were bled by venupuncture of the retro-orbital vein. The rats were screened for haemoparasites using Haematocrit Centrifugation Technique (HCT), Wet Mount Technique (WMT) and Thin Blood Smear (TBS). The Packed Cell Volume (PCV), Total plasma Protein (TPP), and differential white blood cell count were determined. These were to serve as pre-infection values.

\section{Experimental Infection}

Following the development of parasitaemia (20-30 parasites per field) of Trypanosoma brucei, the rats were humanely sacrificed, and $4 \mathrm{mls}$ of blood collected in a graduated beaker containing salts of Ethylene Diamine Tetraacetate (EDTA) as anticoagulant, and diluted with $5 \mathrm{mls}$ of physiological saline solution to attain total volume of $9 \mathrm{mls}$.Using insulin needle each rat from IV, IM, SC and IP groups were inoculated with $0.2 \mathrm{mls}$ each of the diluted blood estimated to contain $1.26 \times 10^{6}$ parasites per ml of blood [7] using IV, IM, SC and IP routes respectively, while the fifth group served as uninfected control. The experimental animals were allowed to pass through the course of the infection.

\section{Collection of Blood Samples}

Blood was aseptically collected from each rat by venipuncture of the retro-orbital vein, $0.1 \mathrm{ml}$ of blood was collected at days 6 and 12 post infection meant for determination of packed cell volume, total plasma protein, total leukocytes, parasitaemia using WMT, TBS and HCT. $0.05 \mathrm{ml}$ of blood was also aseptically collected from each rat by venupuncture of the central caudal vein every two days post infection until the end of the experiment meant for parasitaemia detection and estimation by TBS and WMT.

\section{Parasitaemia Detection and Estimation}

Trypanosomes were detected using wet blood film and parasitaemia were estimated using the rapid "Matching" method described by Herbert and Lumsden [7].

\section{Hematological Examination}

Packed Cell Volume (PCV) and Total White blood cell count (WBC) were determined using the standard microcapillary haemocytometric methods, respectively, as described by Jain 1986).Total plasma protein was determined using a refractometer method (Coulter Electronics, earts England) as described by Kerr [8].

\section{Monitoring}

The parasitemia of infected rats were checked every 3 days intervals post inoculation for 12 days by doing wet mount and HCT. The wet mount was observed under X 40 magnification, and the HTC was observed under XI0 magnification with oil immersion. TBS was done for every positive result and observed under XI00 magnification. PCV was determined at days 6 and 9 post infection using microhematocrit capillary tube, microhematocrit centrifuge and microhematocrit reader to read the result. The experiment lasted for a period of 21 days post infection.

\section{Statistical Analysis}

The values of the PCV, WBC, parasitemia and total plasma protein of each group of the animals were summarized as mean \pm SEM. Variation among mean values was evaluated by variance (ANOVA). Values of $\mathrm{P}<0.05$ were considered statistically significant, version 4.0 of graph pad prism was used for the statistical analysis of data. 


\section{Results}

The results of this study showed that rats (Rattus norvegicus) are susceptible to experimental infection with Trypanosoma brucei by different routes of inoculation with mean pre-patent periods of $3.00 \pm 0.000,4.00 \pm 1.000,7.50 \pm 1.500$ and $9.00 \pm 1.732$ days respectively for the IV, IM, SC, and IP. There was increase in mean parasitaemia for the entire group which was statistically significant. This difference was most for the IV group and least for the IP group $(\mathrm{P}<0.05)$.There was no statistically significant difference in mean $\mathrm{PCV}$ for the entire group, except for the IP group $(\mathrm{P}<0.05)$. There was increase in mean total plasma protein at the initial stage, which decreases at the later stage of infection, but there was no statistical difference for the entire group except for the IP group $(\mathrm{P}<0.05)$. There was slight increase in mean total WBC for the entire, but there was no statistically significant difference between the groups. Mean survival time of infected rats was $15.25 \pm 2.016,13.25 \pm 2.720$, $12.25 \pm 0.250,12.75 \pm 1.250$ for the IV, IM, SC and IP respectively which has no statistical difference between the groups. On TBS done for every positive result, all the three forms of the parasites ( $T$. brucei) i.e slender, intermediate and stumpy forms were observed (Tables 1-5).

Table 1: Mean Parasitemia (Parasites per field) \pm SEM of experimentally infected rats with Trypanosoma brucei via different routes and uninfected control.

\begin{tabular}{|c|c|c|c|c|c|}
\hline Group & Day 0 & Day 3 & Day 6 & Day 9 & Day 12 \\
\hline A & $0.00 \pm 0.000$ & $0.75 \pm 0.479$ & $2.33 \pm 1.202$ & $3.33 \pm 1.202$ & $5.33 \pm 0.667$ \\
\hline B & $0.00 \pm 0.000$ & $0.50 \pm 0.289$ & $2.33 \pm 0.667$ & $3.00 \pm 0.577$ & $4.33 \pm 0.882$ \\
\hline C & $0.00 \pm 0.000$ & $0.00 \pm 0.000$ & $1.00 \pm 0.408$ & $2.33 \pm 0.333$ & $3.33 \pm 0.333$ \\
\hline D & $0.00 \pm 0.000$ & $0.00 \pm 0.000$ & $1.00 \pm 0.408$ & $1.25 \pm 0.479$ & $2.33 \pm 0.333$ \\
\hline UC & $0.00 \pm 0.000$ & $0.00 \pm 0.000$ & $0.00 \pm 0.000$ & $0.00 \pm 0.000$ & $0.00 \pm 0.000$ \\
\hline
\end{tabular}

Key: A = IV, B= IM, C = SC, D = IP, UC = Uninfected control.

Table 2: Mean PCV (\%) \pm SEM of experimentally infected rats with Trypanosoma brucei via different routes and uninfected control.

\begin{tabular}{|c|c|c|c|}
\hline Group & Day 0 & Day $\mathbf{6}$ & Day $\mathbf{1 2}$ \\
\hline A & $49.75 \pm 1.797$ & $44.25 \pm 2.810$ & $45.50 \pm 3.500$ \\
\hline B & $49.00 \pm 1.225$ & $51.25 \pm 2.016$ & $51.00 \pm 1.528$ \\
\hline C & $52.25 \pm 2.995$ & $49.00 \pm 2.708$ & $40.33 \pm 2.963$ \\
\hline D & $49.25 \pm 0.750$ & $42.25 \pm 1.315$ & $39.00 \pm 1.528$ \\
\hline UC & $42.50 \pm 1.756$ & $42.25 \pm 1.315$ & $41.50 \pm 1.190$ \\
\hline
\end{tabular}

Key: A = IV, B= IM, C = SC, D = IP, UC = Uninfected control.

Table 3: Mean TPP (g/dL) \pm SEM of experimentally infected rats with Trypanosoma brucei via different routes and uninfected control.

\begin{tabular}{|c|c|c|c|}
\hline Group & Day 0 & Day 6 & Day 12 \\
\hline A & $6.65 \pm 0.4787$ & $7.75 \pm 0.2500$ & $7.55 \pm 0.6185$ \\
\hline B & $6.90 \pm 0.5447$ & $7.20 \pm 0.3055$ & $6.40 \pm 0.3055$ \\
\hline C & $5.70 \pm 0.2082$ & $6.90 \pm 0.1291$ & $6.33 \pm 0.7688$ \\
\hline D & $6.30 \pm 0.1915$ & $7.00 \pm 0.000$ & $6.47 \pm 0.2667$ \\
\hline UC & $6.40 \pm 0.7439$ & $6.43 \pm 0.6613$ & $6.45 \pm 0.2667$ \\
\hline
\end{tabular}

Key: A = IV , B= IM, C = SC, D = IP, UC = Uninfected control.

Table 4: Mean WBC (X 10\% / L) \pm SEM of experimentally infected rats with Trypanosoma brucei via different routes and uninfected control.

\begin{tabular}{|c|c|c|}
\hline Group & Day 0 & Day 12 \\
\hline A & $3.075 \pm 0.5186$ & $5.367 \pm 1.7950$ \\
\hline B & $3.250 \pm 0.8770$ & $8.050 \pm 2.0500$ \\
\hline C & $5.075 \pm 1.1880$ & $9.400 \pm 6.3740$ \\
\hline D & $3.700 \pm 0.2380$ & $8.333 \pm 2.8620$ \\
\hline UC & $4.000 \pm 0.8124$ & $3.275 \pm 0.2869$ \\
\hline
\end{tabular}

Key: A = IV, B= IM, C = SC, D = IP, UC = Uninfected control.
Table 5: Mean survival time (days) \pm SEM post infection of rats experimentally infected with $T$ brucei via different routes.

\begin{tabular}{|c|c|}
\hline Group & Survival Time Post Infection (Days) \\
\hline A & $15.25 \pm 2.016$ \\
\hline B & $13.25 \pm 2.720$ \\
\hline C & $12.25 \pm 0.250$ \\
\hline D & $12.75 \pm 1.250$ \\
\hline
\end{tabular}

Key: A = IV, B=IM, C = SC, D = IP.

\section{Discussion}

The influence of infective dose was eliminated in this study by inoculating approximately the same number of the parasite [9]. The shortest pre-patent periods are in the IV route group compared to IM, which is shorter than SC route group and shortest in IP route showing that IV group established patent parasitemia earliest, followed by IM group, then SC and latest in IP route group. This could be due to direct inoculation of the infected blood into the vein making the parasites present in the blood some hours post inoculation for the IV group, for the IM route it could be due to major blood vessel in proximity to the muscle injected, but for the SC group it will take more time for the parasites to get into systemic circulation and most time for the IP group. The observed fall in PCV in the infected animals in the present study coincide with patency of infection. This is in agreement with the previous records of Trypanosoma evansi infections [10-12] and it strongly suggests that the presence of live trypanosomes in circulation and the level of parasitemia may be responsible for the development of anemia [13]. Anemia in trypanosome-infected animal is consistent and determines the severity of infection [14]. However, this study showed that, the fall in PCV in the entire group were not statistically significant, except for the IP group. 
The initial rise in total plasma protein seen in all the groups of this study is in accordance with the observations of Gutierrez [11] in Trypanosoma evansi infected camel and Trypanosoma brucei infected rabbits respectively. The increase might be due to hypergammaglulinemia in response to the infection [15]. It is also likely that the increase might have been triggered by the parasites derived intracellular enzymes and proteins derived from lysed parasites by the host immune system. However, the fall in the total plasma protein concentrations seen in the later stages of all the groups except for the uninfected control is in accordance with the report of Shehu, in experimentally Trypanosoma evansi infected goats. The decrease may be suggestive of an increase in protein break down or urea loss and hemodilution. Moreover, there was slight leukocytosis in all the groups except for the uninfected control. This is based on the fact that tolerant animals develop leukocytosis and non-tolerant animals develop leucopenia [16,17].

\section{Conclusion}

This study revealed that the pre-patent period is shortest in rats infected intravenously and longest in those infected intraperitoneally, same applies to parasitaemia, which was highest in intravenous group and lowest in intraperitoneal group. Though the different routes of inoculation of Trypanosoma brucei ensued significant variation in pre-patency, parasitaemia and survival time but not in PCV and Total plasma protein. Route of inoculation may not have significant influence on the severity of infection due to Trypanosoma brucei strain used in this study in albino rats.

\section{References}

1. Maudlin I (2006) "Centennial review: African trypanosomiasis". Annals of Tropical Medicine and Parasitology 100(8): 679-701.

2. Swallow BM (2000) "Impact of Trypanosomiasis on agriculture". PAAT, Technical and Scientific Series 2: 35-38.

3. Simarro PP, Diarra A, Ruiz Postigo JA, Franco JR, Jannin JG (2011) The human African trypanosomiasis control and surveillance programme of the world health organization 2000-2009: The way forward. PLoS Negl Trop Dis 5(2): e1007.

\section{ISSN: 2574-1241}

DOI: $10.26717 / B J S T R .2019 .13 .002465$

Akefe IO. Biomed J Sci \& Tech Res

This work is licensed under Creative Commons Attribution 4.0 License

Submission Link: https://biomedres.us/submit-manuscript.php
4. Chris J Schofield, John Kabayo P (2008) Trypanosomiasis vector control in Africa and Latin America. Parasites \& Vectors 1(24): 1-7.

5. Brun R, Blum J, Chappuis F, Burri C (2009) Human African Trypanosomiasis. Lancet 375(9709): 148-159.

6. Vickerman K (1985) Developmental Cycles and Biology of Pathogenic Trypanosomes. British Medical Bulletin 41(2): 105-114.

7. Herbert WJ, Lumber WHR (1976) Trypanosoma brucei: A rapid 'matching' method for estimation of the host parasitaemia. Exp parasitol 40(3): 427-431.

8. Kerr MG (1989) Chemical Biochemistry and Haematology in: Veterinary Laboratory Medicine. Blackwell Scientific Publications, Oxford p. 1-30.

9. Murray M, Dexter, TM (1988) Anaemia in bovine African trypanosomosis. Acta Trop 45(4): 389-432.

10. Queiroz AO, Capello PH, Jansen AM (2000) Biological and biochemical characteristics of isolates of Trypanosoma evansi from pantanal of matogrosso- Brazil Vet Parasitology 92(2): 107-118.

11. Gutierrez JA, Juste MC, Doreste F, Morales I (2005) An outbreak of abortions and high neonatal mortality associated with Trypanosoma evansi infection in dromedary camel in the Canary Islands. Veterinary parasitology 130(1-2): 163-168.

12. Shehu SA, Ibrahim NDG, Esievo KAN, Mohammed G (2006) Role of erythrocyte surface sialic acid in inducing anaemia in Savannah Brown bucks experimentally infected with Trypanosoma evansi. Vet Arhiv 76(6): 521-530.

13. Holmes PH, Jennings FW (1976) Pathophysiology of parasitic infection. Acad Press New York Pg 199.

14. Anosa OV (1988) Haematological and biochemical changes in human and animal trypanosomosis. Rev Elev Med Vet Pays Trop 41(1): 65-78.

15. Rahman ZU (1992) Serum biochemical, enzyme and haematological changes in one-humped camels infected with sura, proceedings of the first international camel conference $\mathrm{R}$ and $\mathrm{W}$ publication (New market) Limited Dubai pp. 405.

16. Paling RW, Moloo SK, Scott JR, Mcodimba FA, Logan H Enfrey, et al. (1991) Susceptibility of N'dama and Boran cattle to Tse-tse transmitted primary and rechallenge infections with a homologous serodeme of Trypanosoma congolense. Parasite Immunol 13(4): 413-425.

17. Brun R, Blum J, Chappuis F, Burri C (2010) Human African trypanosomiasis. Lancet 375(9709): 148-159.

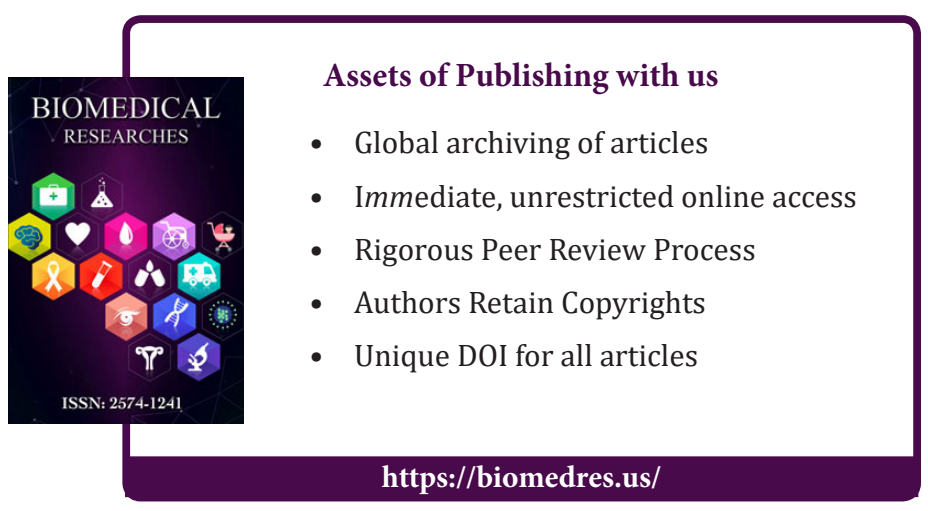

Cite this article: Ameh MP, Jatau ID, Ada G, Akefe IO. Hematological and Biological Changes in Different Routes of Experimental Trypanosoma 